\title{
Les discours à la mi-temps d'entraîneurs de rugby
}

\author{
Alain Mouchet
}

Alain Mouchet.

Résumé. Nous présentons une recherche collaborative visant à rendre intelligible et transformer l'activité d'entraîneurs expérimentés en compétition, plus particulièrement leurs discours à la mi-temps en match de rugby. L'éclairage du cadre théorique techno-psychophénoménologique permet d'étudier l'activité des entraîneurs en contexte écologique. L'approche multi-méthodes qui articule des observations et des entretiens d'explicitation, permet de cerner à la fois les dimensions observables et les logiques subjectives. L'analyse quantitative des interventions a mis en avant un profil communicationnel commun et des particularités liées au contexte. De plus, la triangulation des données a permis d'identifier des savoirs d'action partagés par les entraîneurs et des styles singuliers de coaching, ainsi que des logiques personnelles d'intervention sousjacentes aux comportements observables. Cette étude contribue à documenter l'expérience subjective des entraîneurs, avec des savoirs incorporés et implicites. Elle procure aussi des concepts et outils utiles pour la formation des entraîneurs.

Mots-clés. Discours à la mi-temps, Coaching en compétition, Multi-méthodes, Expérience subjective, Formation des entraîneurs

\section{Introduction}

Les entraîneurs effectuent une activité de coaching durant les matchs, au cours de laquelle il s'agit par exemple d'observer le jeu, de gérer l'opposition entre les équipes, de s'occuper des remplacements des joueurs, de prendre en compte les arbitres, de communiquer en jeu et lors des pauses officielles (Jones, 2006 ; Mouchet \& Bouthier, 2008). Le coaching en match a été peu étudié en sport de haut niveau (Cloes et al., 2009), malgré quelques exceptions (Bouthier \& Durey, 1995 ; Mouchet \& Duffy, 2018 ; Saury \& Durand, 1998), alors qu'il a été identifié comme un élément important dans les fonctions principales des entraîneurs (Lara-Bercial et al., 2017). Ces études ont souligné que le coaching est un processus complexe, dynamique et dépendant du contexte (Lyle, 2002), nécessitant des adaptations à la dynamique évolutive du match et des prises de décisions rapides et efficaces à des moments importants, parfois sous forte pression temporelle et mentale.

Le discours à la mi-temps constitue l'un de ces moments importants. En effet, les entraîneurs de rugby ont un accès limité aux joueurs durant le match en raison des contraintes réglementaires qui les obligent à rester dans une zone technique en dehors du terrain. Le discours à la mi-temps constitue une occasion importante de réguler la stratégie initiale d'avant-match et de communiquer avec les joueurs (Mouchet \& Maso, 2018).

Il y a donc des enjeux importants dans cette étude. L'enjeu scientifique concerne l'investigation fine pour comprendre l'activité en situation écologique, en accédant aux dimensions observables et aux processus sous-jacents. L'enjeu socioprofessionnel concerne l'analyse et la transformation des pratiques des entraîneurs et des joueurs, où la subjectivité est une ressource pour la formation. Au-delà de la performance, c'est aussi un volet éducatif qui est envisagé chez les entraîneurs (Jones, 2006) lors des sessions de formation, et indirectement l'éducation des joueurs leaders dans la prise de responsabilités lors de ces discours.

Pour éclairer l'activité des entraîneurs, nous mobilisons certains aspects de la technologie des Activités Physiques, Sportives et Artistiques (APSA), mis en avant initialement par Bouthier et Durey (1995), et développés ensuite par d'autres auteurs en fonction de leurs objets respectifs (Eloi \& Uhlrich, 2011 ; Mouchet, 2011). Nous utilisons ainsi un mode de pensée systémique pour rendre intelligible l'activité des entraîneurs, en proposant une modélisation 
Les discours à la mi-temps d’entraîneurs de rugby

présentée dans la figure 1 ci-dessous. Nous distinguons trois plans imbriqués d'analyse : le contexte général, le contexte local et la situation (Mouchet, 2018). En l'occurrence la situation correspond ici aux interventions lors du discours à la mi-temps.

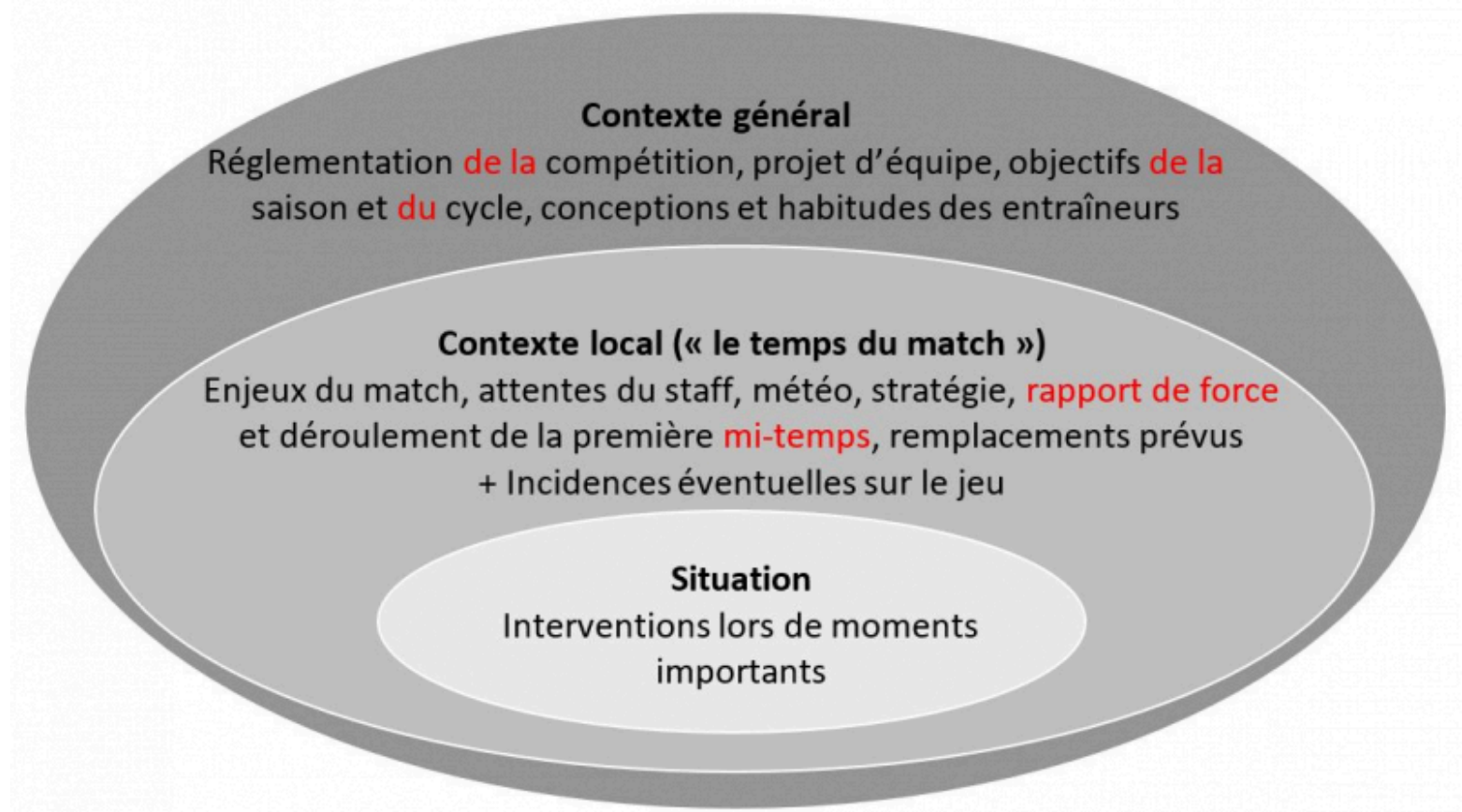

Figure 1 : Approche systémique de l'activité des entraîneurs en match

De plus, cet éclairage de l’approche technologique est articulé avec la psychophénoménologie (Vermersch, 2012) qui permet de rendre intelligible le vécu subjectif dans entraîneurs dans leur discours à la mi-temps, en l'occurrence les actes moteurs et mentaux, l'attention et les prises de décision, soit des aspects intuitifs et provisoirement non conscients durant le discours.

L'objectif de cette étude est de caractériser les compétences d'entraîneurs de rugby en ce qui concerne leur discours à la mi-temps, et de comprendre leur expérience vécue en situation. L'objet de recherche est l'organisation et le contenu du discours, ainsi que les effets sur la production du jeu.

\section{Méthodologie}

\section{Participants et déroulement de l'étude}

L'échantillon concerne huit entraîneurs des équipes U23, U20, U19, U15 d'un club d'élite, l'AS Montferrand. Ils sont âgés de 30 à 50 ans $(m=43)$ et possèdent les diplômes requis. Leur expérience en coaching va de 4 ans à 23 ans $(m=13)$. Quatre entraîneurs travaillent à temps plein et quatre à mi-temps. La méthodologie comprend deux phases interactives, qui alternent des études de cas avec un binôme d'entraîneurs en match, soit deux matchs par équipe, et trois soirées de formation avec tous les entraîneurs du club qui le souhaitent, le directeur du Centre de Formation, un entraîneur de l'équipe professionnelle et les préparateurs mentaux, soit une quinzaine de personnes. Le discours à la mi-temps dans ces catégories dure environ cinq minutes, avec une petite fluctuation selon les matchs. 
Actes de la 11ème Biennale de l'ARIS : Former des citoyens physiquement éduqués. Axe 3 - Au-delà de la performance, 10.25518 /sepaps 20.475

\section{Recueil de données}

Nous avons mis en place un dispositif en spirale, qui alterne et articule six études de cas et trois sessions collectives de formation (Mouchet, 2015 ; Mouchet \& Maso, 2018). Dans les études de cas, nous avons effectué une approche multiméthodes (Mouchet, 2018): (1) un bref entretien semi-dirigé juste avant le match pour identifier avec les entraîneurs la stratégie, les attentes pour le match et les remplacements prévus ; (2) l'enregistrement audiovisuel de l'intervention avec deux caméras et un micro-cravate sur chaque entraîneur; (3) le scénario du match pour documenter le contexte de l'intervention à la mi-temps (score, rapport de force...) et l'influence des consignes sur la production du jeu lors de la deuxième mi-temps ; d) un entretien d'explicitation dans les jours suivants pour explorer le vécu de l'action et favoriser la prise de conscience des actions. Le traitement des données a été effectué par une analyse de contenu pour les entretiens semi-dirigés, une analyse quantitative des communications par l'outil Comerem et le tableau du vécu pour les entretiens d'explicitation (Mouchet, 2018).

En ce qui concerne les sessions de formation, la première session a été consacrée à la présentation et l'exploitation par les entraîneurs, des résultats partiels des deux premières études de cas, à partir de différents supports : extraits de verbatim des discours à la mi-temps, séquences vidéo, extraits de verbatim des entretiens d'explicitation. Elle a permis de dégager les facteurs d'efficacité et les sources de difficultés. La deuxième session a permis de poursuivre ce travail, à partir de la triangulation des données obtenues lors d'études de cas menées avec un autre staff technique. Des tendances communes et des styles personnels de coaching sont ainsi apparus. De plus, une nouvelle demande a émergé de la part des entraîneurs : la volonté de prendre en compte l'intersubjectivité, en étudiant aussi la façon dont les joueurs s'approprient ou non le discours. Trois questions 'flash' en une minute à l'issue du discours ont été posées à cinq joueurs. La dernière session a permis d'affiner l'identification des savoirs d'action partagés ou singuliers, et de cibler l'analyse des résultats relatifs au point de vue des joueurs lors des cinquième et sixième étude de cas.

\section{Résultats}

Tout d'abord, l'analyse quantitative des communications révèle un profil communicationnel commun aux entraîneurs : (1) adressage surtout vers l'équipe ; (2) communications unilatérales ; (3) parler (sans crier) avec une forte gestuelle d'accompagnement ; (4) contenu centré sur stratégico/tactique et sur le mental ; (5) équilibre entre des rétroactions négatives et positives; (6) intentions variées (imposer, convaincre, influencer l'état mental, analyser le jeu) au sein des binômes, selon le match. Des différences ont aussi été notées en lien avec le contexte singulier du match (caractéristiques de l'adversaire, score et météo notamment).

Des savoirs d'action partagés ont été identifiés à partir de l'articulation des études de cas et des sessions de formation, à propos de l'organisation des discours : (1) exploiter des moments clés (concertation préalable au sein du binôme environ 5' avant, minute de récupération, transition entre les deux entraîneurs) ; (2) organiser spatialement le groupe en cercle plus ou moins serré selon le contexte momentané ; (3) structurer le discours avec différentes étapes (capter l'attention des joueurs, organiser le discours avec quelques points clés...) ; (4) utiliser une technicité de communication (congruence du non verbal, ton juste, adressage à des joueurs clés, gestuelle d'accompagnement) ; (5) finir éventuellement par des consignes individuelles après le coup de sifflet de l'arbitre.

Les savoirs d'action partagés sont également relatifs au contenu des discours : (1) prendre en compte le contexte à l'issue de la première mi-temps (score, vent, arbitre, rapport de force) ; (2) structurer le bilan avec des repères récurrents ( $+/-/+$ » ou « ce qui a marché / les menaces adverses / les propositions pour répondre efficacement »); (3) ajuster la charge informationnelle des consignes ; (4) être parfois négatif mais moduler et expliquer ; (5) finir par une motivation et des encouragements ; (6) s'appuyer sur les principes fondamentaux du rugby ; (7) gagner du temps en utilisant de l'implicite partagé dans le projet de jeu collectif. 
Les discours à la mi-temps d’entraîneurs de rugby

Des savoirs d'action singuliers ont également été formalisés, exprimant des styles personnels de coaching ou des adaptations circonstanciées. Nous retenons par exemple : (1) rester debout pour percevoir l'arbitre afin de réguler temporellement l'intervention ou s'accroupir au sein du cercle pour fixer l'attention des joueurs sur un point bas ; (2) questionner différemment (nature, durée, destinataires) selon l'âge et l'éducation de certains joueurs leaders ; (3) laisser un degré d'implication variable à certains joueurs dans le bilan et les perspectives de jeu ; (4) utiliser la vitesse de resserrement du groupe comme indicateur de l'attente ou pas d'informations et comme source d'adaptation du mode d'intervention dans l'instant, avec une intervention plus calme ou plus énergique ; (5) élaborer des routines efficaces personnalisées au sein de chaque binôme et répartir le contenu des interventions.

Notons que lors des études de cas, les entretiens d'explicitation, tout en contribuant à la caractérisation des savoirs d'action indiqués précédemment, ont également mis au jour des savoirs d'expérience implicites. Ainsi, le moment de rassemblement du groupe constitue en même temps l'occasion de rassembler ses idées en triant, synthétisant, clarifiant. La dynamique attentionnelle met en avant l'importance des regards entre entraîneurs et joueurs (révélateurs, catalyseurs, transmetteurs). De plus, les entraîneurs cherchent à la fois à rassurer et se rassurer eux-mêmes dans la relation aux joueurs et au binôme (attitudes, contacts, échanges, rituels). Certains sont aussi fortement occupés par la gestion de leurs propres émotions (diffuser/masquer, les utiliser au service de la communication, se connaître). Enfin, des logiques personnelles d'intervention sous-jacentes aux apparences se dévoilent (buts réels, valeurs, image de soi...). Enfin, en ce qui concerne l'efficacité des discours, les questions flash avec les joueurs ont révélé : (1) une compréhension différenciée selon le statut (titulaire ou remplaçant) ; (2) une appropriation sélective selon leur poste et s'ils étaient directement concernés ou pas ; (3) une sensibilité accrue à quelques mots du capitaine ; d) une interprétation personnelle voire invention de certaines consignes. L'analyse des scénarios du match met en lumière une efficacité des consignes sur le jeu produit en deuxième mi-temps en règle générale, mais un déplacement des problèmes dans d'autres secteurs du jeu, non abordés à la mi-temps, et une diminution de l'influence de ces consignes au fil de la deuxième mi-temps.

\section{Discussion}

Lyle (2002) soulignait que le coaching en match semble souvent lié à l'instinct, aux sensations, à l'expérience, aux décisions intuitives. Les cadres d'analyse et l'approche multi-méthodes présentés dans cette étude, permettent d'adopter une approche holistique pour mieux identifier ces dimensions à la fois observables et subjectives. Nous contribuons ainsi à caractériser les compétences importantes relatives au coaching en compétition (Lara-Bercial et al., 2017), en complétant les travaux sur les conceptions des entraîneurs (Mouchet \& Duffy, 2018) par ce qu'ils font réellement sur le terrain, dans la lignée de travaux antérieurs en rugby (Bouthier \& Durey, 1995 ; Mouchet \& Bouthier, 2008).

Nous rejoignons aussi des travaux antérieurs qui pointent la nature dynamique et complexe du coaching en compétition (Saury \& Durand 1998), ainsi que sa dépendance aux différents niveaux de contexte tels que nous les avons identifiés, du plus englobant au plus précis : «général », « local », « situationnel » (Mouchet, 2018). Entre prévisions et régulations in situ lors de la mi-temps, les entraîneurs élaborent des routines de coaching que l'on peut assimiler à un genre technique, enraciné dans la culture d'entraînement du rugby français comme macro-système, tout en étant imprégné par le système sociotechnique du club considéré, avec sa culture locale. Ces routines de coaching concernent la préparation de l'intervention, l'organisation et le contenu du discours. Elles constituent un facteur d'efficacité pour gérer la pression temporelle et l'incertitude relative sur la durée de la mi-temps. Néanmoins nous avons repéré chez certains entraîneurs des difficultés à adapter ces routines en fonction d'évènements imprévus qui perturbent leur mise en œuvre. Enfin, les sessions de formation ont permis d'identifier des axes de travail personnalisé : améliorer l'observation du jeu et l'analyse des problèmes pour préparer, structurer et accroître la pertinence du discours ; optimiser le temps de concertation préalable et utiliser la minute de récupération pour se concentrer ; sensibiliser les 
joueurs à leur implication (auto-évaluation, rôle des leaders...) ; mettre à profit l'intervention du binôme pour être attentif aux joueurs et ajuster son intervention si nécessaire.

\section{Conclusion}

Des savoirs d'action partagés et des styles individuels de coaching ont été identifiés, ainsi que des savoirs d'expérience plus tacites. Cette recherche collaborative a réactualisé au sein du club des débats sur la gestion temporelle, le rôle des joueurs leaders et du capitaine, la gestion des remplacements, la pertinence de l'observation du jeu sous-jacente au discours, la technicité à l’œuvre dans les discours.

Dans ce dispositif en spirale, nous retrouvons l'enchaînement de l'action, du réfléchissement des pratiques, de la réflexion partagée sur des matériaux conscientisés et un retour à l'action. Le vécu individuel dans une situation passée fait ainsi écho, ou encore fait l'objet d'une résonance avec les problématiques personnelles des autres participants qui ont pu vivre des situations similaires. La formation des entraîneurs est alors une occasion privilégiée d'analyse de leur pratique, de partage d'expérience, d'utilité sociale de la recherche et aussi éducation des joueurs leaders à l'autonomie et aux responsabilités.

\section{Bibliographie}

Bouthier, D., \& Durey A. (1995). La compétence d'un entraîneur de rugby. Éducation permanente, 123, 65-79.

Cloes, M., Lenzen, B., \& Trudel, P. (2009). Analyse de la littérature francophone portant sur l'intervention de l'entraîneur sportif, publiée entre 1988 et 2007. STAPS, 1(83), 7-23.

Eloi, S., \& Uhlrich, G. (2011). La démarche technologique en STAPS : analyse conceptuelle et mise en perspective pour les sports collectifs. eJRIEPS, 23, 20-45.

Jones, R.L. (2006). The sports coach as educator: re-conceptualising sports coaching. Oxon: Routledge.

Lara-Bercial, S., North, J. Hämäläinen, K., Oltmanns, K., Minkhorst, J., \& Petrovic, L. (2017). European Sport Coaching Framework. Champaign, Illinois, United States: Human Kinetics.

Lyle, J. (2002). Sports coaching concepts: A framework for coaches' behaviour. London: Routledge.

Mouchet, A. (2011). Les registres de technicité : Un concept utile pour analyser l'activité des sujets dans les APSA ? eJRIEPS, 23, 76-92.

Mouchet, A. (2015). L'explicitation au cœur d'un dispositif de formation en spirale qui articule vécu singulier et expérience collective. Recherche et formation, 80, 91-106.

Mouchet, A. (2018). L'expérience subjective en recherche et en formation. Villeneuve d'Ascq : Presses Universitaires du Septentrion.

Mouchet, A., \& Bouthier, D. (2008). Le coaching des sélections nationales en rugby. In P. Fleurance, \& S. Pérez (Eds.), Interrogations sur le métier d'entraîneur : interroger les entraîneurs au travail ? Revisiter les conceptions qui organisent l'entraînement pour repenser le métier d'entraîneur(e) (pp.281-288). Les Cahiers de l'INSEP, 39, Paris : INSEP Publications.

Mouchet, A., \& Duffy, P. (2018). Rugby coaches' perceptions of their in-competition role. Sport Coaching Review, 9(1), 24-47. 
Les discours à la mi-temps d'entraîneurs de rugby

Mouchet, A., \& Maso, F. (2018). Subjective lived experience: a resource for coaches' education. LASE Journal of Sport Science, 9(1), 60-77.

Saury, J., \& Durand, M. (1998). Practical knowledge in expert coaches: on-site study of coaching in sailing. Research Quarterly for Exercise and Sport 69(3),254-266. doi: 10.1080/02701367.1998.10607692

Vermersch, P. (2012). Explicitation et phénoménologie. Paris : PUF.

PDF automatiquement généré le 2023-04-26 15:07:59

Url de l'article : https://popups.uliege.be/sepaps20/index.php?id=475

Publié par ULiège Library en Open Access et distribué suivant les termes et les conditions de la licence CC-BY (https://creativecommons.org/licenses/by/4.0/deed.fr) 